\title{
The expanding and contracting roles of the genome in regulation of the ovarian reserve
}

\author{
David F. Albertini ${ }^{1}$
}

Published online: 17 August 2016

(C) Springer Science+Business Media New York 2016

In the lands of plenty, having products in excess or of ready availability for consumption contributes to a sense of satisfaction envied by the have-nots. While reproductive medicine continues to tip the balance in favor of fecundity-for-all, one of the most challenging rate-limiting factors in the daily practice of human ARTs remains that of the ovarian follicle reserve. Whether allowed to proceed with advancing age, or accelerated in response to genetic predispositions or environmental insults, the depletion of ovarian follicles spells the end of natural fecundity as we know it today.

Confronting the reality of follicle loss across the human reproductive lifespan is a serious matter for patients and physicians alike. And as much as hopes are raised with the introduction of technologies like gene editing (see meeting summary by LaBarbera in this issue [1]) for altering transmissible traits, classical genetic approaches have uncovered a bewildering array of genomic entities potentially involved in the establishment and maintenance of the ovarian reserve.

Among the many candidate genes topping the list of possible conspirators (or facilitators depending on one's perspective) are those involved with the synthesis and/or secretion of FSH subunits and their respective receptor and signal transducing elements [2], the gene products of which have been implicated in a wide range of female reproductive conditions

Capsule The factors that specify, maintain, and eventually conscribe to effect extinction of the ovarian follicle reserve have been elusive and bemusing at a genetic level, but interestingly not so at the gene product level, as the search for the mechanisms underscoring the programmed or pathologically precocious depletion of the ovary's most formative compartment continues.

David F. Albertini

dalbertini@thechr.com

1 The Center for Human Reproduction, New York, NY, USA from age at onset of menopause to polycystic ovary syndrome (PCOS). Next in line, and popular today as a sentinel for the ovarian reserve, is the granulosa cell specific gene product anti-mullerian hormone (AMH), one of a number of genes in the family of transforming growth factors (TGFbetas) that by virtue of their intra-ovarian patterns of gene expression, fine tune the process of folliculogenesis at various steps along the pathway to ovulation.

When it comes to genetic predisposition to disease, the fragile X mental retardation (FMR1) gene comes immediately to mind given its relationship to the risk of premature ovarian failure (POF) in women harboring a permutation load of CGG trinucleotide repeats $[3,4]$. Such trinucleotide repeat expansions in the FMR1 gene situated at Xq27.3 come in various sizes that may compromise protein (FMRP) production in the case of the full mutation or yield protein products whose role in the etiology of POF or neurological conditions associated with Fragile X syndrome (FXS) remains a matter of speculation. In this issue of JARG, Noto and colleagues provide a thorough treatment of this subject [5].

Of the matters arising in this important area of research are first principle association studies regarding the varying degrees of expansion and the relationship of genotypes to reproductive phenotypes, as considered in the paper from the group of Benadiva at the University of Connecticut Health Center [6]. As the discourse evolves over how linear modifications in DNA structure at specific genes ties into both transcriptional efficiency and the downstream impact of altered protein products, the larger questions attracting attention for disorders known to have a basis in mutations of the expansion variety are being directed at the genomic landscape and gene positioning factors in terms of both genetic and epigenetic contributions. While this broader perspective of how gene activation and repression takes hold (something that undoubtedly will require revisiting and re-educating a generation or two used 
to the Mendelian mindset), drawing conclusions regarding the genetic control of the ovarian follicle reserve remains an exercise in futility despite its relevance to emerging areas such as fertility preservation.

It is in this context that Barad and coworkers review the topic of ovarian reserve and consider the aging ovary as a moving target that can compound interpretation of many of the studies done to date on the putative linkage between FMR1 and POF [7]. They introduce the issue of redundancy in relation to the functional ovarian reserve and point out that our efforts to use traditional tools of genetic analysis impose typically unrecognized limitations that for FXS will influence not only our understanding of the basic mechanisms underlying disease but the ways in which genetic counseling will direct the critical decisions made by patients carrying mutations.

We close this month drawing our readers' attention to the article by $\mathrm{Lu}$ and $\mathrm{Lv}$ and their colleagues, which provides further coverage on the evolving topic of genetic analyses in human embryos embracing a future perspective that is soon to include the ability to evaluate single cells [8]. As always, we welcome your feedback on how to best serve our readership, and in keeping with our international commitment to draw upon experts in reproductive medicine, we are pleased to welcome to our editorial board Drs Adriana Bos-Mikich and Paula Navarro from Brazil and Dr. Soren Ziebe from Denmark.

\section{References}

1. LaBarbera AR. Proceedings of the International Summit on Human Gene Editing: a global discussion-Washington, D.C., December 13, 2015. J Assist Reprod Genet. 2016. doi:10.1007/s10815-0160753-x.

2. Ruth KS, Beaumont RN, Tyrrell J, Jones SE, Tuke MA, Yaghootkar $\mathrm{H}$, et al. Genetic evidence that lower circulating FSH levels lengthen menstrual cycle, increase age at menopause and impact female reproductive health. Hum Reprod. 2016;31(2):473-81.

3. Mailick MR, Hong J, Greenberg J, Smith L, Sherman S. Curvilinear association of CGG repeats and age at menopause in women with FMR1 premutation expansions. Am J Med Genet B Neuropsychiatr Genet. 2014;165B(8):705-11.

4. Pastore LM, Johnson J. The FMR1 gene, infertility, and reproductive decision-making: a review. Front Genet. 2014;5:195.

5. Noto V, Harrity C, Walsh D, Marron K. The impact of FMR1 gene mutations on human reproduction and development: a systematic review. J Assist Reprod Genet. 2016. doi:10.1007/s10815-0160765-6.

6. Maslow BL, Davis S, Engmann L, Nulsen JC, Benadiva CA. Correlation of normal-range FMR1 repeat length or genotypes and reproductive parameters. J Assist Reprod Genet. 2016. doi:10.1007 /s10815-016-0732-2.

7. Barad DH, Kushnir VA, Gleicher N. The importance of redundancy of functional ovarian reserve when investigating potential genetic effects on ovarian function. J Assist Reprod Genet. 2016. doi:10.1007/s10815-016-0762-9.

8. Lu L, Lv B, Huang K, Xue Z, Zhu X, Fan G. Recent advances in preimplantation genetic diagnosis and screening. J Assist Reprod Genet. 2016. doi:10.1007/s10815-016-0750-0. 